\title{
Investigation of failure mechanisms in silicon based half cells during the first cycle by micro X-ray tomography and radiography ${ }^{\#}$
}

\author{
Fu Sun ${ }^{a, b, *}$, Henning Markötter ${ }^{b}$, Kang Dong ${ }^{a, b}$, Ingo Manke $^{b}$, Andre Hilger ${ }^{b}$, Nikolay \\ Kardjilov ${ }^{b}$ and John Banhart ${ }^{a, b}$ \\ ${ }^{\mathrm{a}}$ Institute of Material Science and Technologies \\ Technical University Berlin \\ 10623 Berlin, Germany \\ ${ }^{\mathrm{b}}$ Helmholtz Centre Berlin for Materials and Energy \\ Hahn-Meitner-Platz 1 \\ 14109 Berlin, Germany \\ *Corresponding Author: fu.sun@helmholtz-berlin.de \\ \# published in: Journal of Power Sources 321, 174-184 (2016) \\ Elsevier, ISSN: 0378-7753 — doi: 10.1016/j.jpowsour.2016.04.126
}

Two proof-of-concept batteries were designed and prepared for X-ray microtomography and radiography characterizations to investigate the degradation mechanisms of silicon (Si) based half cells during the first cycle. It is highlighted here for the first time that, apart from the significant volume expansion-induced pulverization, the electrochemical "deactivation" mechanism contributes significantly to the capacity loss during the first charge process. In addition, the unexpected electrochemically inactive $\mathrm{Si}$ particles are also believed to substantially decrease the energy density due to the inefficient utilization of loaded active material. These unexpected findings, which cannot be deduced from macroscopic electrochemical characterizations, expand the inherent explanations for performance deterioration of Si-anode material based lithium ion batteries (LIBs) and emphasize the vital value of microscopic techniques in revealing the correlation between macroscopic electrode structure and the overall electrochemical performance.

\section{Introduction}

Following their commercial introduction in the early 1990s, lithium ion batteries (LIBs) have penetrated ubiquitously into the market for energy storage systems, e.g. laptops and mobile phones [1, 2]. More recently, advanced LIBs with a larger specific energy, higher power density and longer cycle life have been considered for powering clean electric vehicles (EVs) and plug-in hybrid vehicles (PHVs), as well as for the storage and distribution of energy from sustainable sources, such as solar and wind energy [3-6]. The key to fabricate such nextgeneration LIB is to exploit high-performance electrode materials. From the material's point of view, silicon (Si) is a promising candidate for the anode of LIBs because it possesses the highest theoretical capacity of $3579 \mathrm{mAh} / \mathrm{g}$ when lithiated to $\mathrm{Li}_{21} \mathrm{Si}_{5}$, that is about 10 times larger than the currently commercialized carbonaceous anode, which features $372 \mathrm{mAh} / \mathrm{g}$ for $\mathrm{LiC}_{6}$ [7-9]. This promising potential of the high specific capacity has spurred considerable investigations of $\mathrm{Si}$ thin films [10], carbon mixed [11, 12] and metal coated [13] $\mathrm{Si}$ composites, along with various Si nanostructures, such as nanoparticles [14], nanowires [15], nanospheres [16] and nanotubes [17] that could serve as building blocks for high-performance anodes. Despite these extensive efforts, Si-based anode LIBs still inevitably suffer from 
substantial capacity decay during lithium insertion and extraction and thus they are now below the requirements for practical applications.

To shed light on the underlying degradation mechanisms, a range of investigation techniques such as scanning electron microscopy (SEM) [18], transmission electron microscopy (TEM) [19], X-ray diffraction (XRD) [20], nuclear magnetic resonance (NMR) [21] and Raman spectroscopy [22] have been adopted. As a result, our knowledge of the intrinsic behaviour of Si upon lithium insertion and extraction has been significantly enhanced [23] and various possible explanations for the performance deterioration have been proposed. For instance, it has been discovered that, apart from the loss of the lithium inventory and electrolyte/binder decomposition [24], the preferential volume expansion along Si [110] directions is as large as $\sim 300 \%$ during lithium insertion and this extraordinary volume change has been proposed as the primary factor contributing to the fatal capacity decay [25]. Nevertheless, prior analytical tools are inherently limited. For example, most of the measurements abovementioned explore the degradation mechanisms on the atomic or single nanoparticle level, overlooking the interplays amongst a multitude of particles and the interactions between the active material and the conductive/binder agent. What is more, a particular battery design, such as open structure and specialized electrolyte that does not adequately simulate the real battery operating conditions is widely employed. On the other hand, most conventional tools only specialize in revealing the structural and compositional information without imaging capability, lacking effective spatially resolved information about the degradation mechanisms. In parallel, some investigations are carried out ex situ, i.e. post-mortem, as opposed to in situ characterizations of dynamic processes. Considering that a realistic composite electrode includes an assembly of ensembles of active particles, an organic polymeric binder and a conducting agent, the ability to probe the dynamic deterioration mechanisms on a multiparticle electrode level is of technological and practical importance. Therefore, it is crucial and emergent that fundamental research techniques are highly needed to further promote the exploration of electrode degradation.

Recently, X-ray imaging based on either laboratory X-ray or synchrotron X-ray sources has rapidly evolved into a powerful characterization tool in materials science [26-42]. Specifically, $\mathrm{X}$-ray imaging has enabled researchers to obtain unprecedented insights into LIBs nondestructively on a length scale ranging from particles to entire electrodes and has contributed markedly to our understanding [29-33]. The pioneering research of employing synchrotron Xray tomography by Ebner et al. features a direct observation and quantification of electrochemical and mechanical degradation in a SnO anode [34]. Meanwhile, Gonzalez et al. have visualized the expansion of large Si particles during the first lithiation step in threedimensions (3D) by means of tomography based on a laboratory X-ray source [35].

Herein, by employing both laboratory X-ray tomography and radiography, we re-inspect the underlying mechanism of performance degradation of Si based half cells from the perspective of entire electrodes, which implies spanning the length scales from individual active particles to the macroscopic electrode ensemble. On the one hand, X-ray microtomography grants us the ability to track the structural evolution induced by volume changes in three dimensions. On the other hand, in operando micro X-ray radiography enables us to directly observe the changes of the active particles and an entire electrode during lithium insertion and extraction. By the combinatorial X-ray tomography and radiography, for the first time we highlight that, apart from the huge volume expansion/contraction of Si particles during cycles, the striking inhomogeneous lithiation/delithiation mechanism amongst ensemble active particles observed in an electrode, which cannot be easily detected in conventional electrochemical measurements and, the unprecedented electrochemical "deactivation" of original electrochemical active particles are another two key factors contributing to the substantial 
performance degradation. This study expands the inherent explanations for performance deterioration of Si-anode material based LIBs, and the new sights would open new design principles and opportunities for high-capacity electrode materials for next-generation energy storage systems.

\section{Experimental Section}

\section{Materials:}

Silicon was received from Elkem AS, Norway. Polyvinylidene difluoride (PVDF) binder, carbon black, Celgard separator, CR2032 coin cells and lithium were purchased from MTI Cor. USA. N-methyl pyrrolidone solvent (NMP) and $1 \mathrm{M} \mathrm{LiPF}$ in a volume-ratio (1:1) mixture of ethylene carbonate (EC) and dimethyl carbonate (DMC) were purchased from Sigma Aldrich. Titanium foil is from ANKURO Int. GmbH, Germany. The housing of proofof-concept beamline battery is made of polyamide-imide (Torlon), from McMaster-Carr company.

\section{Characterization:}

Scanning electron microscopy (SEM) image were taken using a Zeiss ultraplus microscope. The electrochemical characterizations were conducted by using an Ivium CompactStat station, Iviumtechnology.

\section{Battery Preparation:}

The composite electrode is made of slurries with weight ratios of Si:carbon black:binder of 75:15:10 in NMP. For the tomo-cell, the slurry was first sandwiched between two glasses and put into an oven at $60{ }^{\circ} \mathrm{C}$ to dry and form a block, which was then cut into small pieces with a razor blade and put directly onto the top of the screws. The resultant Si composite electrode was a small piece of $1.7 \times 1.7 \times 0.2 \mathrm{~mm}$ (length $\times$ width $\times$ height). Before the Si composite was assembled into the tomo-cell, it was weighed by a digital balance and the amount of $\mathrm{Si}$ particles was determined from the origian mass ration. The Si composite electrode mass in the tomo-cell was around $0.9 \mathrm{mg}$. For the radio-cell, the slurry was casted onto the $5 \mu \mathrm{m}$ thick titanium (Ti) foil. The area of the slurry was around $4 \times 3 \mathrm{~mm}$ (length $\mathrm{x}$ width). To remove the NMP, the casted $\mathrm{Ti}$ foil was also dried in an oven at $60{ }^{\circ} \mathrm{C}$. Before and after the casting process, Ti foil was weighted to determine the weight of the electrode materials. The Si composite in the radio-cell was around $1 \mathrm{mg}$. These two proof-of-concept batteries were assembled in an argon filled glovebox with humidity and oxygen levels below $0.1 \mathrm{ppm}$. During battery assembly, these two cells were only hand-assembled without exerting extra pressures. For the tomo-cell, metallic lithium (2.5 mm diameter, $1 \mathrm{~mm}$ thick) was placed on top of the other screw, and served as a counter and reference electrode. For the radio-cell, a lollipop shape copper foil without inner region (out diameter is $10 \mathrm{~mm}$ and inner diameter is 6 $\mathrm{mm}$ ) was used as a current collector and the Ti foil was placed on top of it. Metallic lithium (6 mm diameter, $1 \mathrm{~mm}$ thick) was used as the opposite electrode. A polymer separator was placed between the lithium electrode and the Si electrode in both cells. After filling these two cells with sufficient electrolyte, they were sealed off and then taken out of the glovebox. For a further comparison of the electrochemical activity of Si composite electrode in the two proofof-concept cells, commercial CR2032 coin-cell with the same Si electrode was assembled and tested.

\section{Micro X-ray Tomography \& Radiography Characterizations:}

$\mathrm{X}$-ray tomography was conducted on the tomo-cell in the original state and after the voltage had dropped to $0.03 \mathrm{~V}, 0.02 \mathrm{~V}, 0.01 \mathrm{~V}$ and $0 \mathrm{~V}$ during discharge. During the discharge process, 
the current was set to be $0.04 \mathrm{~mA}$. The five tomographies are implied by alphabet a, b, c, d and e in Fig. 4, Fig. 5 and Fig. 6. The particular parameters for the X-ray tomography are as follows: the energy is $60 \mathrm{kV}$, the current is $166 \mu \mathrm{A}$, the source detector distance (SDD) is 500 $\mathrm{mm}$, the source object distance (SOD) is $58 \mathrm{~mm}$. Given the resulting magnification, each detector pixel therefore represented $5.76 \mu \mathrm{m} /$ voxel in the sample. The exposure time is $2.1 \mathrm{~s}$. During the $360^{\circ}$ rotation, 800 projections were recorded. During the charge process, the current was switched to $0.01 \mathrm{~mA}$ and the tomography was conducted after the voltage had risen to $0.5 \mathrm{~V}, 0.7 \mathrm{~V}, 1 \mathrm{~V}, 1.5 \mathrm{~V}$ and $2 \mathrm{~V}$. The five tomographies are implied by alphabet $\mathrm{f}, \mathrm{g}$, h, i, and j in Fig. 4, Fig. 5 and Fig. 6. The locations of the particles shown in Fig. 3, Fig. 4 and Fig. 5 are marked in SI Fig. 4. More information is given in the Supporting Information (SI).

For the X-ray radiography measurements, characterization was conducted in situ during the whole discharge/charge process of the radio-cell. During the discharge process, the current was $0.07 \mathrm{~mA}$ for $0-18 \mathrm{~h}$ and then was switched to $0.04 \mathrm{~mA}$ for the rest of discharge process. During the charge process, the current was set to be $0.015 \mathrm{~mA}$ for $6.5 \mathrm{~h}$ and then was changed to $0.01 \mathrm{~mA}$ until the voltage was $2 \mathrm{~V}$. The radio-cell was facing the $\mathrm{X}$-ray source during the characterization.

Data Processing:

To keep the validity of the measurements, only a median filter $(1 \times 1 \times 1)$ was performed in ImageJ. 3D particle visualization was performed by VGStudio MAX. Analysis of the particles is conducted by Imaje J. More information is given in the SI.

\section{Results and Discussion}

X-ray microtomography and radiography was conducted with a laboratory X-ray CT system [43]. To be fully compatible with X-ray imaging and at the same time to completely represent working LIBs, we have designed and manufactured two dedicated proof-of-concept electrochemical cells: a cell for tomography (tomo-cell) and a cell for radiography (radio-cell). Fig. 1 displays photographs and the corresponding schematic illustrations of the two proof-ofconcept batteries as well as a schematic representation of the X-ray micro CT setup [44]. 
(a)

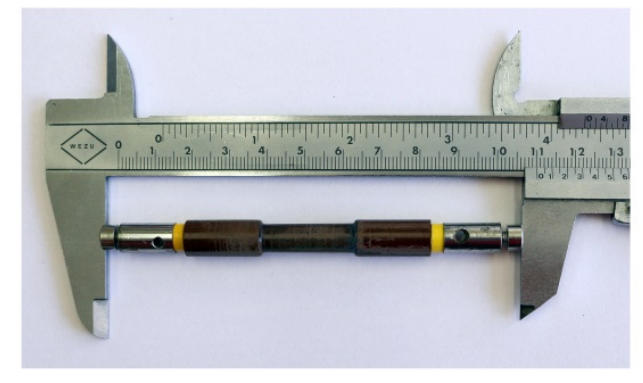

(c)

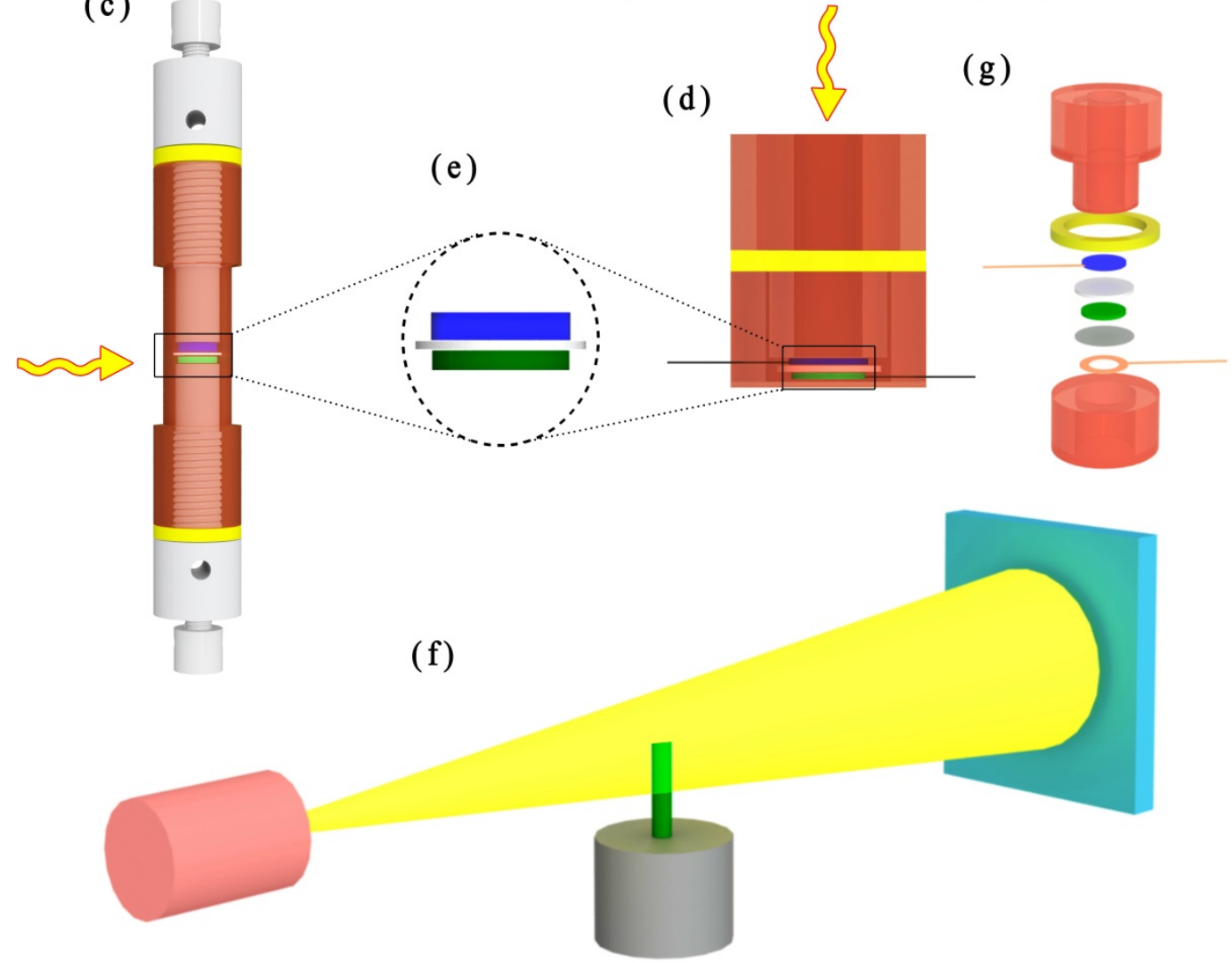

(b)

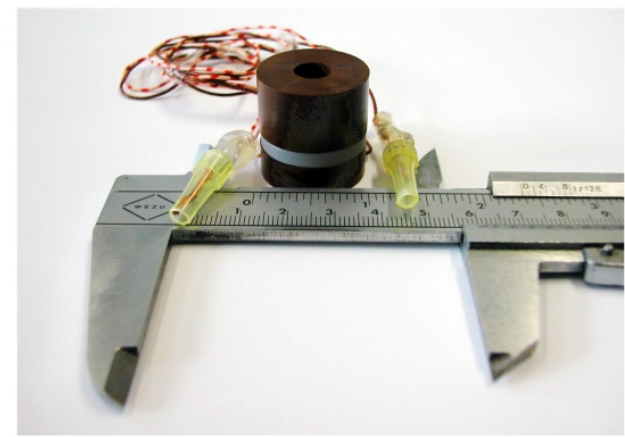

(d)

g) b) radio-cell. c) Corresponding schematic representation of the tomo-cell. d) and g) Corresponding schematic representations of the radio-cell. e) Enlarged region of interest comprising from top to bottom: lithium (blue), separator (grey) and electrode material (green). g) Schematic illustration of the radio-cell, from top to bottom are, the upper housing (brown), sealing ring (yellow), lithium plate (blue) with copper wire, separator (white), $\mathrm{Si} /$ carbon/binder composite (green), titanium foil current collector (gray), lollipop-shaped copper current collector (copper), the lower housing (brown). f) Schematic representation of the X-ray micro CT setup. From left to right: X-ray source (red), cone X-ray beam (yellow), sample representing either of the two cells (green) and rotation table (grey), detector (blue). The waves in c) and d) represent the direction of X-rays.

After assembling the two proof-of-concept batteries, cyclic voltammetry (CV) was performed to verify the reduction and oxidation characteristics of Si. The insets of Fig. 2 show the CV curves of the two batteries scanned at rate of $1 \mathrm{mV} \mathrm{s}^{-1}$ in the potential window of $0-2.5 \mathrm{~V}$. Although there are parasitic redox peaks (arrows in CV figure) associated with contamination and/or electrolyte decomposition [45, 46], the clearly visible cathodic peak above $0 \mathrm{~V}$ and an anodic peak at $0.5 \mathrm{~V}$ are related to the $\mathrm{Li}$ alloying and de-alloying with $\mathrm{Si}$, respectively. The 
results are in good agreement with previously reported Si/Carbon composites [9, 47], thus confirming the electrochemical reactions inside our proof-of-concept batteries.

Then we conduct the X-ray imaging investigations by galvanostatically discharge/charge these two electrochemical batteries: for tomography, the discharge/charge process was stopped during the tomographic data acquisition and continued afterwards (i.e. the measurement is quasi in situ). For radiography, the measurement is continuously conducted simultaneously during the discharge/charge process of the radio-cell (i.e. imaging is truly in situ). The discharge/charge curves of both cells, showing the typical characteristic feature of Si are displayed in Fig. 2 and they are in good agreement with previous results [9]. Note that for the tomographic imaging, a newly assembled battery is used, while for radiographic imaging, the battery is first used to obtain the CV scans and then directly used for in situ radiography measurement (read more in Supporting Information (SI)). For a further comparison of the electrochemical activity of Si materials in the two proof-of-concept cells, commercial coin-cell assembled with the same Si electrode was conducted and the resultant discharge/charge curve is shown in SI Fig. 1. It is found that these three cells have a low coulomb efficiency during the first charge and the reason may stem from the significant particle pulverization of the large Si particles currently used [19].

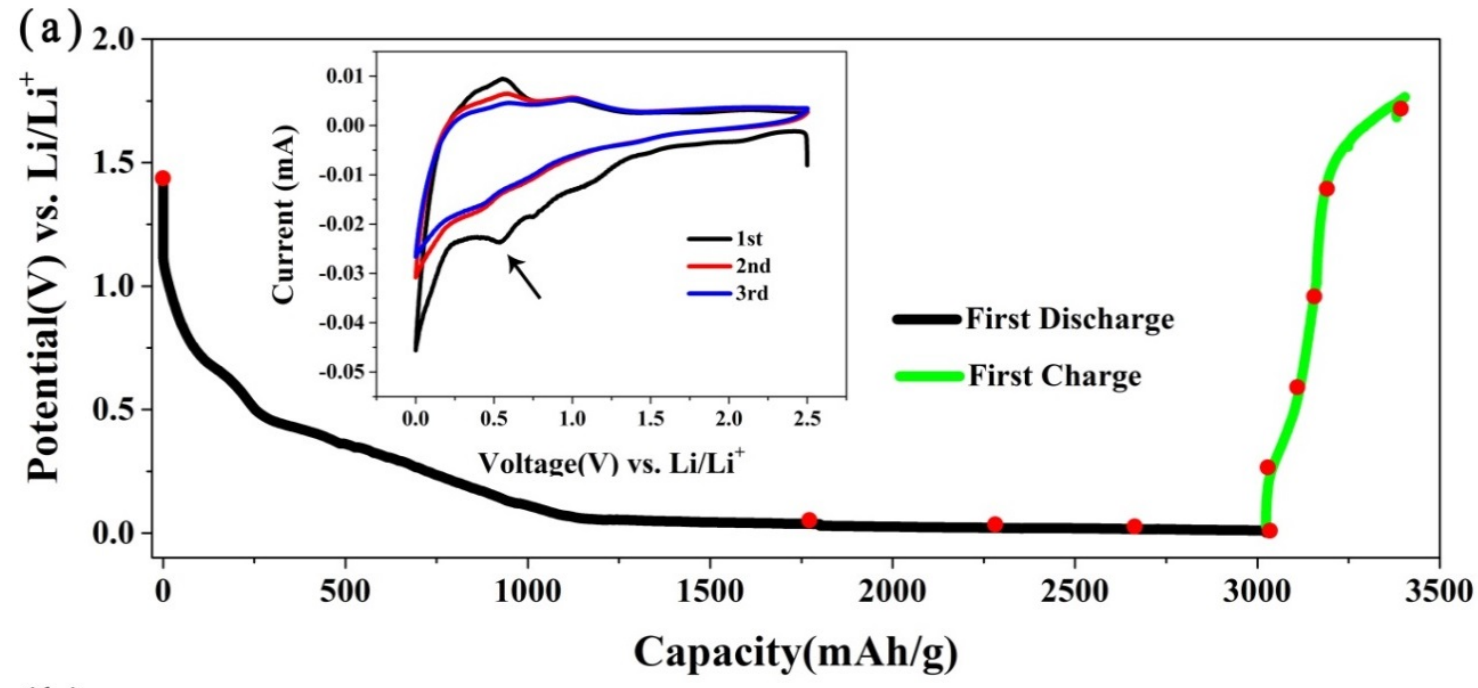

(b)

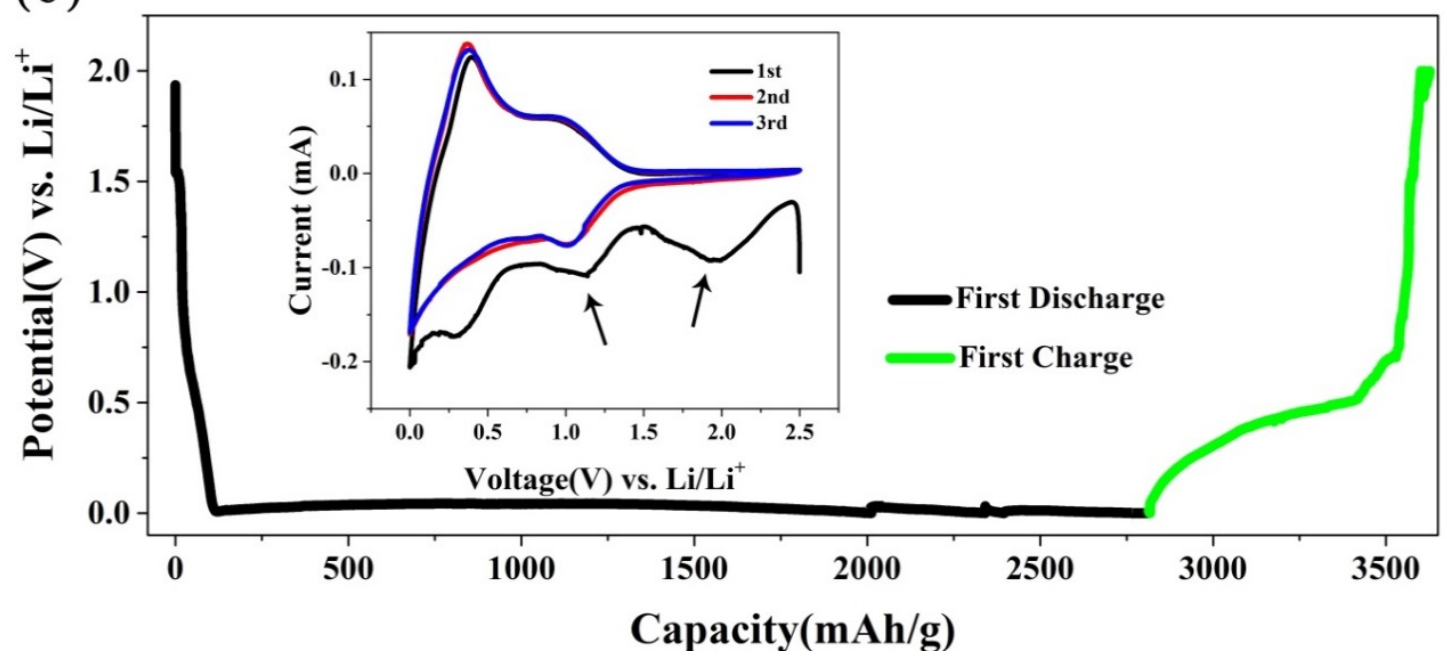

Fig. 2. Electrochemical characterization of the tomo-cell and radio-cell: a) the first discharge-charge curve of the tomo-cell, the inset shows the CV curves; b) same as a) for the radio-cell. 
Micro-computed X-ray imaging is an emerging analytical technique that measures variations in X-ray attenuation of samples and it is particularly well suited to track changes from particles morphological evolution to electrode architecture changes over time as characterizations can be conducted in situ and non-destructively [48]. Consequently, previously unprecedented degradation mechanisms are highlighted here for the first time. In the present work, a distribution of micron sized Si particles ranging from 125 to $180 \mu \mathrm{m}$ in diameter are used for both tomo-cell and radio-cell because of the renewed interest in using micron sized particles as commercial LIBs [49]. To begin with, 3D visualization of Si particles evolution within an operating LIB during the first discharge/charge process is presented by micro X-ray tomography. First, the pristine state of the freshly prepared tomocell is tomographically recorded with 800 projections covering an angular range of $360^{\circ}$ with $2.1 \mathrm{~s}$ exposure time. Then the battery is galvanostatically discharged to $0.03 \mathrm{~V}$ [35]. At this point, the discharge process is stopped and the $2^{\text {nd }}$ tomography is conducted. After this, the discharge is continued and followed by a $3^{\text {rd }}$ tomography. Altogether 10 tomograms during the first discharge/charge process are made (5 during the discharge and 5 during the charge). All the tomograms conducted during the first discharge/charge process are implied by red spots in Fig. 2a and all the parameters are the same. Fig. 3a and Fig. 3b show projection images of the Li electrode|separator|Si electrode assembly within the proof-of-concept batteries. While the Li electrode and the porous plastic separator appear nearly invisible due to their low X-ray absorption coefficients, the Si electrode is clearly visible. Fig. 3c shows a grayscale slice after reconstruction of the tomo-cell and Fig. 3d an enlarged part of the radiocell. In order to qualitatively validate our X-ray imaging techniques, scanning electron micrographs (SEM), as shown in Fig. 3e, are given to compare with the reconstructed raw grayscale slice images and the radiography images. The unambiguous agreement indicates that the X-ray imaging technique accurately captures the morphology of Si electrode.

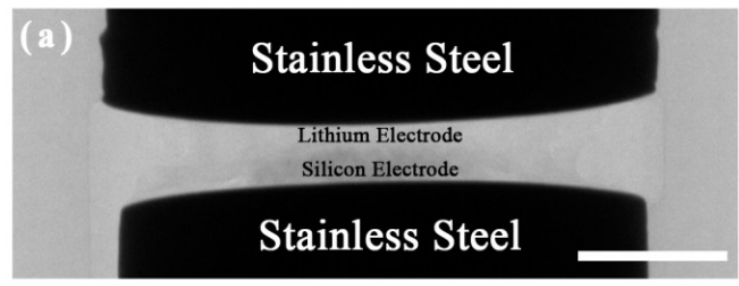

(c)
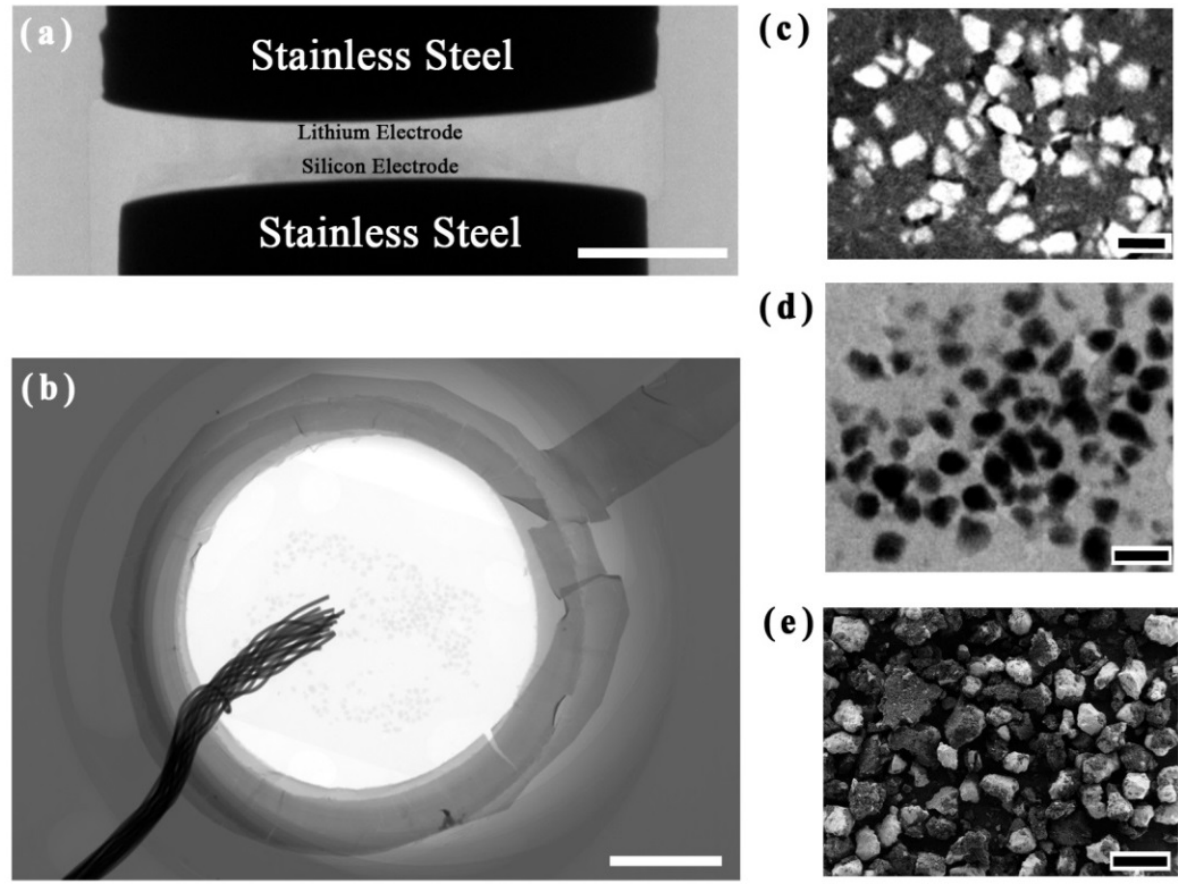

(d)

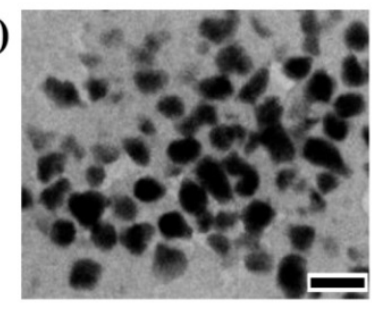

(e)

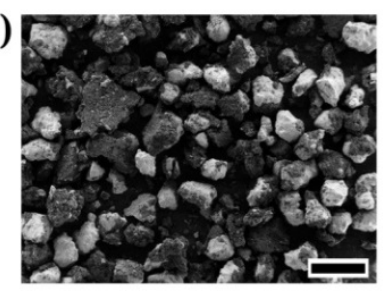

Fig. $3 \mathrm{X}$-ray imaging results of the assembled Li electrode|separator|Si electrode: a) X-ray projection image of the tomo-cell, the scale bar is $1 \mathrm{~mm}$. b) 2D radiography of the radiocell, the scale bar is $2 \mathrm{~mm}$. c) Example of a reconstructed slice image of the tomo-cell. d) Cutout of a radiographic image of the radio-cell. e) Scanning electron micrograph of the mixed Si electrode material. The scale bar in c-e) are all 200 $\mu \mathrm{m}$. 
We now track in detail the morphological changes during lithium insertion and extraction of one randomly chosen particle, illustrating other electroactive particles evolution as a function of discharge/charge. Fig. 4 shows a series of cross-sectional slices taken from tomographies of the Si particle in different stages of discharge/charge. The corresponding 3D rendering of this particle is displayed in SI Fig. 1. Histograms of the attenuation coefficients in sub volumes containing the particle are displayed as a function of discharge/charge process see Fig. $4 \mathrm{k}$ and Fig. 4l. The leftmost peak corresponds to the weakly absorbing carbon black, binder and electrolyte surrounding the particle. Feature changes to the rightmost peak are directly related to the evolution of the active Si particle. Prior to the electrochemical reduction, the attenuation coefficient histogram for the particle consists of one peak located gray level of 6000. During lithium insertion, see Fig. 4k, along with the magnitude of this peak progressively decreases and moves to the left, the particle evolves gradually into a weakly Xray attenuating material. This implies that the particle has completely transformed from the high-density Si phase to a low-density $\mathrm{Li}_{\mathrm{X}} \mathrm{Si}$ phase $(1<\mathrm{x}<4.4)$. Meanwhile, a notable volume expansion to around $200 \%$ (compared with pristine state) is observed in the fully lithiated state. These results are in good agreement with previous results [19, 35]. During lithium extraction, see Fig. 4l, along with the attenuation peak increases and shifts towards the original direction, we observe a homogeneous increase in attenuation coefficient. However, it is worth noting that, neither the peak position nor the peak shape of Si is restored to the original state by the end of the first charge process. This may stem from incomplete delithiation and/or significant trapping of lithium by the electrolyte decomposed byproducts [50], which is considered as one of the reasons of the performance decay during the first cycle.
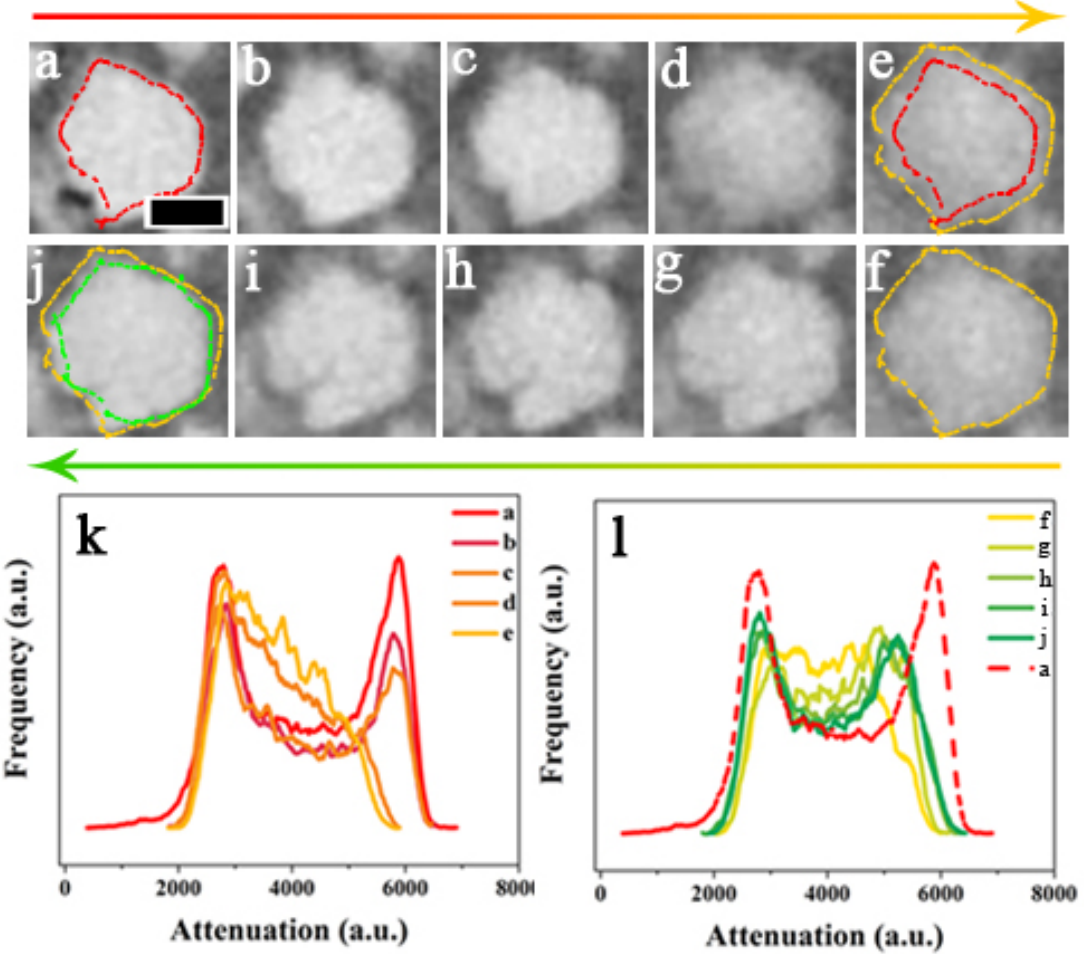

Fig. 4 Evolution of an electrochemically active Si particle: a)-e), tomographic slices through the particle during the first discharge step (see arrow direction); f)-j), tomograms of the first charge process (see arrow direction). k) and l), attenuation coefficient histograms of the specific particle as a function of discharge and charge (dashed red line is the pristine state). Outline of the pristine state (red outline in a)) and outlines of the discharged state (yellow outline in e)) and charged state (green outline in j)) are shown for visual comparison. The scale bar in a) is $100 \mu \mathrm{m}$ long and is the same in all the images. 
Surprisingly, we have also detected an unprecedented phenomenon that many electroactive Si particles become electrochemically inactive during the first delithiation process. This electrochemically deactivation progress is illustrated in Fig. 5, in which, the electroactive particle originally effectively participates in the discharge process, from Fig. 5a to Fig. 5e, but turns to be inactive during the subsequent charge process, contributing to the capacity loss during the first charge process, as we can clearly see the unchanged shape of the particle, from Fig. 5f to Fig. 5j, and the stationary attenuation profile during delithiation, Fig. $5 \mathrm{l}$. Another unexpected phenomenon is that several $\mathrm{Si}$ particles never undergo lithiation/delithiation during the discharge/charge process, i.e., they are electrochemically inactive during the whole macroscopic battery level lithium insertion and extraction process, as shown in Fig. 6. On the whole, from an entire electrode-scale point of view, as displayed in SI Fig. 4, we can unambiguously observe that some electrochemically active Si particles undergo a continuous phase transformation during macroscopic battery level discharge/charge, some electrochemically active Si particles undergo an electrochemical deactivation process after the lithium insertion and some non-electroactive Si particles are completely inactive during the first cycle even as the voltage drops to zero against $\mathrm{Li}^{+} / \mathrm{Li}$. In addition, we also observe that some Si particles emerge and/or disappear in the same slice as a function of discharge/charge process. These distinctive phenomena clearly imply that apart from the large volume expansion during lithiation/delithiation, there are many other factors that contribute to the ultimate performance deterioration in Si-based LIBs.
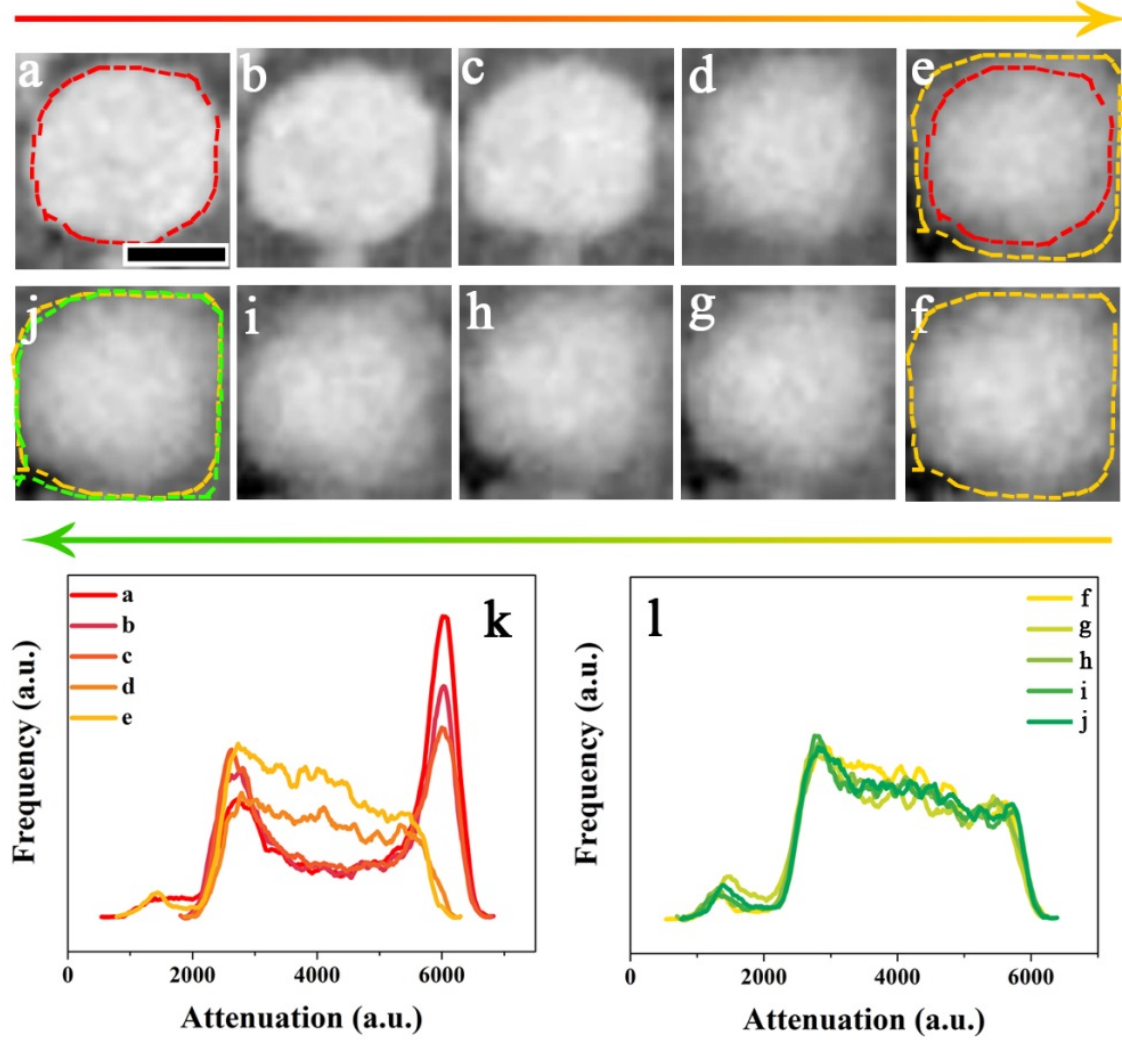

Fig. 5. Evolution of electrochemical deactivation of a Si particle: a)-e), tomographic slices during the first discharge step (see arrow direction); f)-j), tomographic slices during the first charge step (see arrow direction). k) and l), attenuation coefficient histograms during discharge and charge, respectively. Outline of the pristine state (red outline in a)) and outlines of the discharged state (yellow outline in e)) and charged state (green outline in j)) are shown for visual comparison. The scale bar is $100 \mu \mathrm{m}$ long and applies to all the images. 

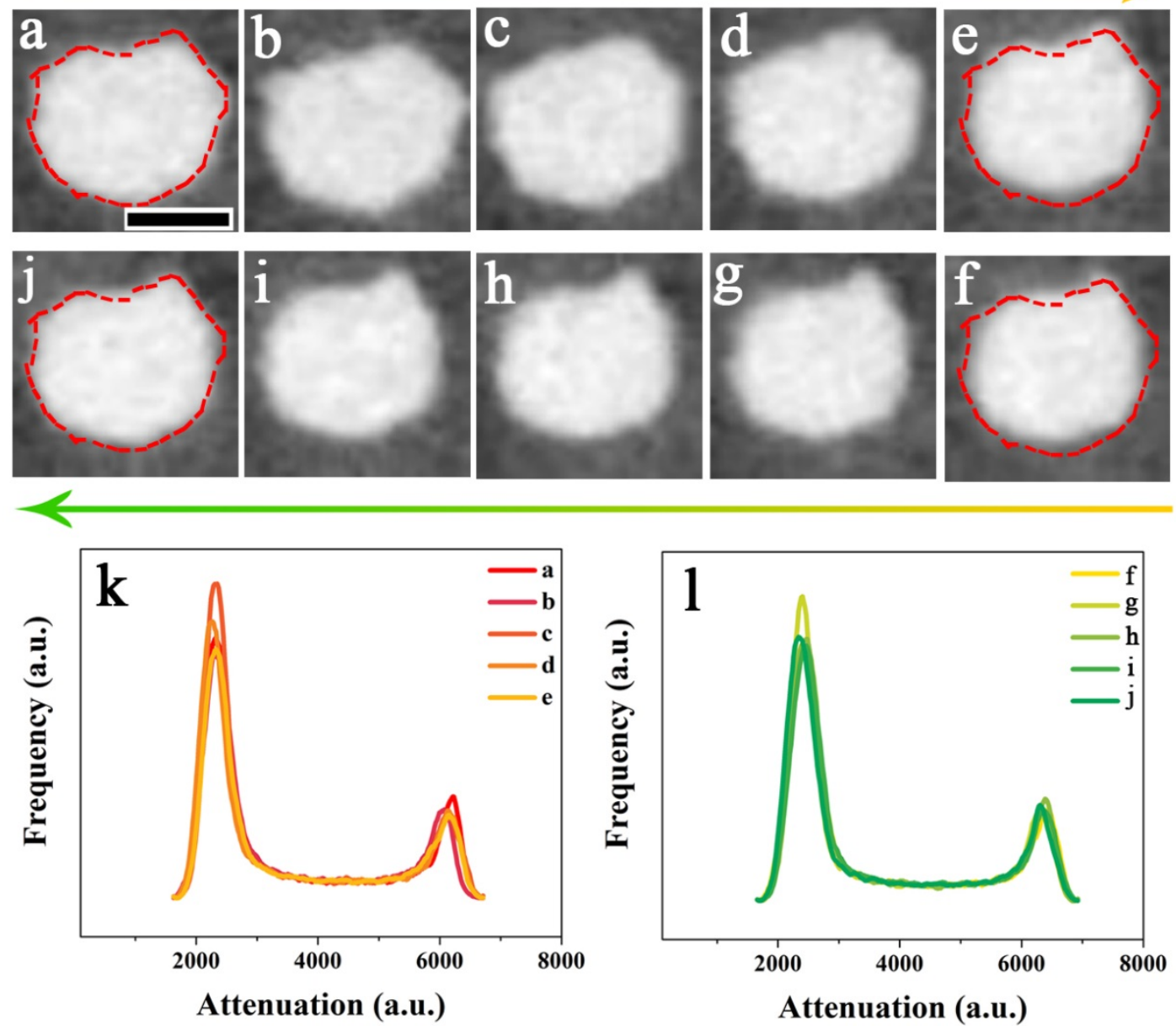

Fig. 6 Evolution of the electrochemically non-active Si particles: a)-e), tomographic slices of the first discharge process (see arrow direction); f)-j), tomographic slices of the first charge process (see arrow direction). k) and l), attenuation coefficient histograms as a function of discharge and charge, respectively; Outline of the pristine state (red in a)) is duplicated to the discharged state e) and charged state j) for visual comparison. The scale bar is $100 \mu \mathrm{m}$.

Moreover, in operando 2D X-ray microradiography provides more sophisticated temporal information within the LIB. As shown in Fig. 7, we can clearly observe the expansion/contraction of the whole electrode material (dotted blue contours from D01 to C10), the expansion and contraction of the electrochemically active particles (green panel), the deactivation phenomenon of originally electroactive Si particles (red panel) and a few $\mathrm{Si}$ particles that are not electrochemically active throughout the whole discharge/charge process (yellow outlines from D01 to C10). Furthermore, the "core-shell" model reaction is clearly observed during the discharge/charge process (see the evolution of particle from Fig. 7a to 7j) [8]. The significant volume expansion-induced pulverization (see the green panel) may lead to electric disconnection of active particles from current collectors [25]. These results are in good agreement with the 3D X-ray microtomography and the whole in operando discharge/charge process is presented in a movie in SI. 


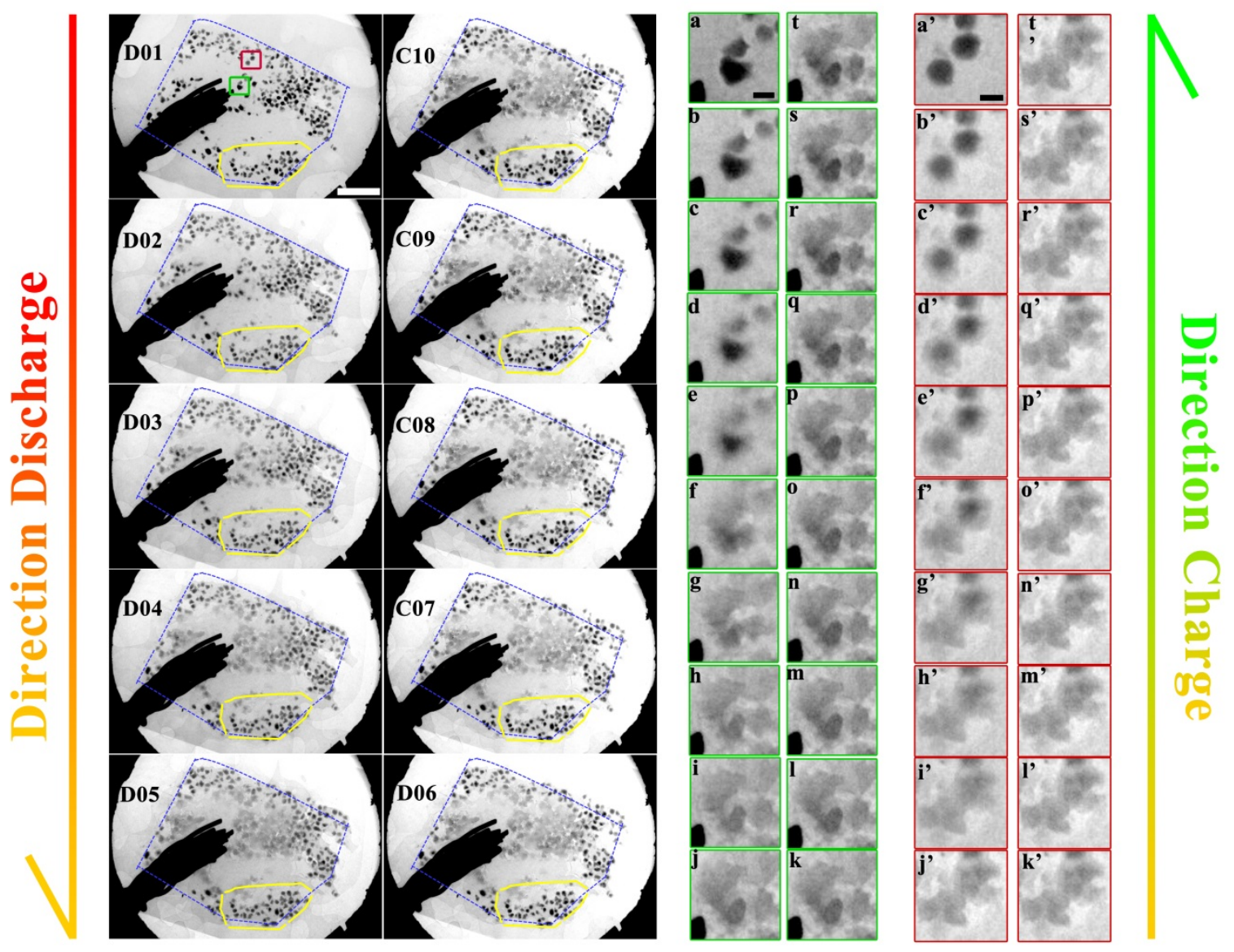

Fig. 7 In situ radiographic characterization of the radio-cell: D01 to C10 represent stages in the first discharge (D) and charge (C) step. The region encircled in blue is the original area that covers the electrode material, increasing numbers refer to elapsing time. The region in green boxes represents the evolution of an electrochemical active Si particle from a to $t$ (the copper lead is seen at the bottom left of each frame). The region in red boxes displays the evolution of electrochemical deactivation from a' to $t$ '. The region encircled in yellow contains many electrochemically inactive particles. The scale bar in D01 is $1 \mathrm{~mm}$ long, that in the small boxes $100 \mu \mathrm{m}$. More information is provided in SI.

Fundamentally speaking, LIBs operate through the reversible insertion into or removal of lithium from electroactive host materials, respectively [51]. The ability of the materials to accommodate the changes associated with the chemical phase transformations that accompany the variations in lithium concentration determines the electrode's utility and battery performance. From an electrochemical point of view, lithium insertion or extraction process entails $\mathrm{Li}^{+}$and $\mathrm{e}^{-}$simultaneously [52]. Contrary to proposed idealised electrochemical models and simulation that [53] electrochemically driven phase conversion is homogeneous and isotropic, such conditions are hardly satisfied in a real commercial LIB due to the complex electrode structures (ensemble of active materials, conductive agents and binders) and the complicated morphology and conditions with respect to porosity, tortuosity, conductivity and percolation ability for the electrolyte [54]. In fact, increasingly more attention is being paid to the investigation of local electrochemical reactions and their relationship with electrochemical performance on a macroscopic and electrode performance $[55,56]$. For example, experimentally a discrepancy is observed between electrochemical measurements that represent the overall state of a cell and spectroscopic data that reflect the local state $[57,58]$. In addition, the heterogeneous local depth of discharge (DOD) and the non-uniform local current distribution have been directly demonstrated by Zhang et al. [59, 60] 
and Ng et al. [61] respectively, by customizing multiple working electrode LIBs. Similarly, significant inhomogeneity of local DOD in different locations of a commercial LIB have been directly detected by Cai et al. [62] and Paxton et al. [63] through neutron diffraction and energy-dispersive X-ray diffraction. Moreover, by using $\mu$ m-resolved Raman spectroscopy, Nanda et al. [64] presented visually the local "spectroscopic" electrochemical variations on an LIB electrode. Finally, recent modeling investigations by Zhao et al. [65] show a direct correlation between energy density and the non-uniformity in local current distribution, demonstrating a potential gain as high as $40 \%$ in energy density through an improved current distribution. Apparently, these locally non-uniform electrochemical reactions, current distributions, ionic/electric conductivity within electrodes will impact on battery performance in a variety of ways, including reduced energy and power, underutilization of capacity, localized heat generation and overcharge or over-discharge of active materials $[56,66]$.

As in our case, it are these electrochemically active Si particles which are ionically and electronically accessed by the electrolyte and connected to the conducting network that directly dictate battery performance. In other words, the total electrode discharging/charging current concentrates only on the electrochemically active Si particles, as clearly shown in Fig. 4, SI Fig. 4 and Fig. 7. These electrochemically active Si particles undergo lithium insertion and extraction to store and release energy, evidenced by the X-ray attenuation coefficient change. During lithium uptake and release, these electroactive Si particles undergo notable volume expansion accompanied by the generation of substantial compressive and tensile stresses [67]. The mechanical forces generated will drive local displacement and rearrangement of other Si particles within the composite electrode, leading to the phenomena that some Si particles emerge and some disappear in one and the same slice, as shown in SI Fig. 4 and Fig. 7. On the other hand, the observed "deactivation" mechanism of originally electrochemically active Si particles probably has three sources: First, an electric contact disconnection. Under the influence of the mechanical forces generated, a lot of displacement/rearrangement of the particles occurs with numerous losses or variations in the contacts. Taking into account that in a stack of particles the electrons are transferred between particles by hopping or tunneling with the contact resistivity depending exponentially on the gap between the contacts, and that a gap of 8-10 nm makes a contact electronically insulating [68], it is plausible to say that the deactivation mechanism is attributed to electric disconnection. The second possibility is the insulating crystalline $\mathrm{Li}_{2} \mathrm{O}$ oxide layer. By using in situ TEM, He et al. report that upon initial Si lithiation, the formed crystalline $\mathrm{Li}_{2} \mathrm{O}$, the product of $\mathrm{Li}$ reaction with the native silicon oxide layer, will partially insulate the particles during subsequent delithiation cycle [69]. The third possible reason is the unshrinkable conductive matrix. Weker et al. proposed that upon the delithiation, when the particles begin to contract, the conductive matrix does not necessarily shrink back to fill the space created by the contracting particles, thus leading to the deactivation of, in their case, germanium particles [70]. Right now we cannot identify a single dominant factor. However, special focus in the future work should be placed to eliminate this phenomenon due to the resultant substantial capacity losses. It is also worth noting that the unexpected electrochemically inactive Si particles within the electrode. From the fact that they are electrochemically inactive from the beginning, it is assumed these Si particles are disconnected into locations of ionically or electronically insulating islands during the electrode preparation, for example binder redistribution during drying, calendaring, cutting and compacting [52]. To get a further quantitative analysis of the influence of different types of Si particles on the obtained discharge/charge capacity, we investigated 78 particles in the same slice in tomo-cell with diameters larger than $50 \mu \mathrm{m}$, as shown in SI Fig. 6. It was found that $13 \%$ (10) of the Si particles were electrochemically in-active through the first cycle, 87\% (68) of the Si particles experienced the first lithiation process and only 24\% (19) of the Si particles experienced the 
first delithiation process. Assuming the theoretical capacity of $\mathrm{Si}$ is $3500 \mathrm{mAh} / \mathrm{g}$, the obtained firstly discharged capacity (3000 $\mathrm{mAh} / \mathrm{g}$ that is around $85 \%$ of the theoretical capacity ) and the obtained firstly charged capacity $(500 \mathrm{mAh} / \mathrm{g}$ that is around $14 \%$ of the theoretical capacity) agree with the results from the quantitative analysis (fraction of $87 \%$ and $24 \%$ (within error consideration)). More information can be found in SI.

The unexpected electrochemical "deactivation" mechanism and the presence of many electrochemical inactive Si particles are alarming because they cannot be easily characterized by conventional macroscopic electroanalytical characterization techniques and should draw attentions from electrode engineers and simulation experts. From an electrode engineer's point of view, the electrode architecture engineering optimization is crucially important. On the one hand, in addition to focusing only on individual particle features to develop nextgeneration LIBs, more and more efforts should be put in optimizing the entire electrode architecture, which can involve all active material particles ionically and electronically connecting to electrolyte and electric conducting network to maximize the utilization of active materials. On the other hand, new conductive/binder agents are highly needed. The matrix of carbon/binder is supposed to sustain the active material's expansion/contraction during cycles as well as to provide an efficient dynamic electric/ionic conducting pathway even under significant electrode transformation. Currently, some exploratory research of developing selfhealing polymer [49] and electric and ionic conductive polymer [71] is underway. From a simulation expert's point of view, in order to develop an electrochemical model that can be used to gain insight into internal processes, to predict performance and operation and optimize cell design, the homogeneous and flawless idealized microstructure characteristics should be compromised with the real complex composite electrodes.

\section{Conclusions}

In summary, we re-explore the mechanisms of dynamic deterioration of Si anode LIBs on an electrode scale by employing X-ray imaging tomography and radiography and for the first time highlight that, apart from the significant volume expansion-induced pulverization and electric disconnection from current collectors, electrochemical "deactivation" contributes significantly to the capacity loss during the first charge process. In addition, the presence of a notable number of electrochemically inactive Si particles is also believed to substantially decrease energy density due to the inefficient utilization of loaded active materials. These unexpected findings, which cannot be obtained by macroscopic electrochemical characterizations and conventional structural/compositional characterizations, provide us with novel insights into the mechanisms of performance degradation of Si anode LIBs. From practical point of view, commercially oriented researches into the local microscopic electrochemical reactions could be motivated and aroused as it governs directly the energy density and capacity retention of a real LIB. And more attention should be paid to the further investigation of the correlation between macroscopic electrochemical performance and local behavior of active materials, to guide the selection and optimization of electrode materials and the manufacture of the electrode.

\section{Supporting Information}

Supporting Information is available in the online version or from the author.

\section{Acknowledgements}

We thank Norbert Beck for fabricating the beamline battery and Elkem AS for providing us with Si particles. This work was sponsored by the Helmholtz Association and the China Scholarship Council. 


\section{References}

[1] A. Barai, G.H. Chouchelamane, Y. Guo, A. McGordon, P. Jennings, J Power Sources, 280 (2015) 74-80.

[2] B. Sun, K. Huang, X. Qi, X. Wei, J. Zhong, Adv Funct Mater, 25 (2015) 5633-5639.

[3] D.X. Liu, J. Wang, K. Pan, J. Qiu, M. Canova, L.R. Cao, A.C. Co, Angew Chem Int Ed, 53 (2014) 9498-9502.

[4] B. Wang, X. Li, B. Luo, L. Hao, M. Zhou, X. Zhang, Z. Fan, L. Zhi, Adv Mater, 27 (2015) 15261532.

[5] F. Sun, K. Huang, Y. Liu, T. Gao, Y. Han, J. Zhong, Appl Surf Sci, 266 (2013) 300-305.

[6] F. Sun, K. Huang, X. Qi, T. Gao, Y. Liu, X. Zou, J. Zhong, Ceram Int, 40 (2014) 2523-2528.

[7] M. Gu, Y. Li, X. Li, S. Hu, X. Zhang, W. Xu, S. Thevuthasan, D.R. Baer, J.-G. Zhang, J. Liu, C. Wang, ACS Nano, 6 (2012) 8439-8447.

[8] J.M. Yuk, H.K. Seo, J.W. Choi, J.Y. Lee, ACS Nano, 8 (2014) 7478-7485.

[9] F. Sun, K. Huang, X. Qi, T. Gao, Y. Liu, X. Zou, X. Wei, J. Zhong, Nanoscale, 5 (2013) 85868592.

[10] N. Suzuki, R.B. Cervera, T. Ohnishi, K. Takada, J Power Sources, 231 (2013) 186-189.

[11] T.H. Hwang, Y.M. Lee, B.-S. Kong, J.-S. Seo, J.W. Choi, Nano Lett, 12 (2012) 802-807.

[12] A. Gohier, B. Laïk, K.-H. Kim, J.-L. Maurice, J.-P. Pereira-Ramos, C.S. Cojocaru, P.T. Van, Adv Mater, 24 (2012) 2592-2597.

[13] Y. He, X. Yu, Y. Wang, H. Li, X. Huang, Adv Mater, 23 (2011) 4938-4941.

[14] H. Kim, B. Han, J. Choo, J. Cho, Angew Chem Int Ed, 47 (2008) 10151-10154.

[15] C.K. Chan, H. Peng, G. Liu, K. McIlwrath, X.F. Zhang, R.A. Huggins, Y. Cui, Nat Nano, 3 (2008) 31-35.

[16] Y. Yao, M.T. McDowell, I. Ryu, H. Wu, N. Liu, L. Hu, W.D. Nix, Y. Cui, Nano Lett, 11 (2011) 2949-2954.

[17] L.-F. Cui, L. Hu, J.W. Choi, Y. Cui, ACS Nano, 4 (2010) 3671-3678.

[18] S.W. Lee, M.T. McDowell, J.W. Choi, Y. Cui, Nano Lett, 11 (2011) 3034-3039.

[19] X.H. Liu, L. Zhong, S. Huang, S.X. Mao, T. Zhu, J.Y. Huang, ACS Nano, 6 (2012) 1522-1531.

[20] J. Li, J.R. Dahn, J Electrochem Soc, 154 (2007) A156-A161.

[21] K. Ogata, E. Salager, C.J. Kerr, A.E. Fraser, C. Ducati, A.J. Morris, S. Hofmann, C.P. Grey, Nat Commun, 5 (2014).

[22] S. Ramdon, B. Bhushan, S.C. Nagpure, J Power Sources, 249 (2014) 373-384.

[23] B. Key, M. Morcrette, J.-M. Tarascon, C.P. Grey, J Am Chem Soc, 133 (2011) 503-512.

[24] E. Sarasketa-Zabala, F. Aguesse, I. Villarreal, L.M. Rodriguez-Martinez, C.M. López, P. Kubiak, J Phys Chem C, 119 (2015) 896-906.

[25] X.H. Liu, Y. Liu, A. Kushima, S. Zhang, T. Zhu, J. Li, J.Y. Huang, Adv Eng Mater, 2 (2012) 722-741.

[26] H.A. Bale, A. Haboub, A.A. MacDowell, J.R. Nasiatka, D.Y. Parkinson, B.N. Cox, D.B. Marshall, R.O. Ritchie, Nat Mater, 12 (2013) 40-46.

[27] J. Moosmann, A. Ershov, V. Weinhardt, T. Baumbach, M.S. Prasad, C. LaBonne, X. Xiao, J. Kashef, R. Hofmann, Nat. Protocols, 9 (2014) 294-304.

[28] T. dos Santos Rolo, A. Ershov, T. van de Kamp, T. Baumbach, Proc Natl Acad Sci, 111 (2014) 3921-3926. 
[29] P.R. Shearing, N.P. Brandon, J. Gelb, R. Bradley, P.J. Withers, A.J. Marquis, S. Cooper, S.J. Harris, J Electrochem Soc, 159 (2012) A1023-A1027.

[30] J. Wang, Y.-c.K. Chen-Wiegart, J. Wang, Angew Chem Int Ed, 53 (2014) 4460-4464.

[31] Y.-c.K. Chen-Wiegart, R. DeMike, C. Erdonmez, K. Thornton, S.A. Barnett, J. Wang, J Power Sources, 249 (2014) 349-356.

[32] J. Wang, ChemElectroChem, 1 (2014) 329-331.

[33] S. Komini Babu, A.I. Mohamed, J.F. Whitacre, S. Litster, J Power Sources, 283 (2015) 314-319.

[34] M. Ebner, F. Marone, M. Stampanoni, V. Wood, Science, 342 (2013) 716-720.

[35] J. Gonzalez, K. Sun, M. Huang, J. Lambros, S. Dillon, I. Chasiotis, J Power Sources, 269 (2014) 334-343.

[36] M. Ebner, F. Geldmacher, F. Marone, M. Stampanoni, V. Wood, Adv Eng Mater, 3 (2013) 845850.

[37] L. Zielke, T. Hutzenlaub, D.R. Wheeler, I. Manke, T. Arlt, N. Paust, R. Zengerle, S. Thiele, Adv Eng Mater, 4 (2014) 1301617.

[38] L. Zielke, C. Barchasz, S. Waluś, F. Alloin, J.C. Leprêtre, A. Spettl, V. Schmidt, A. Hilger, I. Manke, J. Banhart, R. Zengerle, S. Thiele, Scientific Reports, 5 (2015) 10921.

[39] L. Zielke, T. Hutzenlaub, D.R. Wheeler, C.-W. Chao, I. Manke, A. Hilger, N. Paust, R. Zengerle, S. Thiele, Adv Eng Mater, 5 (2015) 1401612.

[40] P.R. Shearing, L.E. Howard, P.S. Jørgensen, N.P. Brandon, S.J. Harris, Electrochem Commun, 12 (2010) 374-377.

[41] A. Haibel, I. Manke, A. Melzer, J. Banhart, J Electrochem Soc, 157 (2010) A387-A391.

[42] I. Manke, J. Banhart, A. Haibel, A. Rack, S. Zabler, N. Kardjilov, A. Hilger, A. Melzer, H. Riesemeier, Appl Phys Lett, 90 (2007) 214102.

[43] O. Hampe, H. Franke, C.A. Hipsley, N. Kardjilov, J. Müller, J Morphol, 276 (2015) 564.

[44] A.R. Benetti, J. Jacobsen, B. Lehnhoff, N.C.R. Momsen, D.V. Okhrimenko, M.T.F. Telling, N. Kardjilov, M. Strobl, T. Seydel, I. Manke, H.N. Bordallo, Sci. Rep., 5 (2015).

[45] C. Pereira-Nabais, J. Światowska, A. Chagnes, F. Ozanam, A. Gohier, P. Tran-Van, C.-S. Cojocaru, M. Cassir, P. Marcus, Appl Surf Sci, 266 (2013) 5-16.

[46] M. Nie, D.P. Abraham, D.M. Seo, Y. Chen, A. Bose, B.L. Lucht, J Phys Chem C, 117 (2013) 25381-25389.

[47] X. Zhou, Y.-X. Yin, L.-J. Wan, Y.-G. Guo, Adv Eng Mater, 2 (2012) 1086-1090.

[48] D.S. Eastwood, V. Yufit, J. Gelb, A. Gu, R.S. Bradley, S.J. Harris, D.J.L. Brett, N.P. Brandon, P.D. Lee, P.J. Withers, P.R. Shearing, Adv Eng Mater, 4 (2014) 1300506.

[49] C. Wang, H. Wu, Z. Chen, M.T. McDowell, Y. Cui, Z. Bao, Nat Chem, 5 (2013) 1042-1048.

[50] S.P.V. Nadimpalli, V.A. Sethuraman, S. Dalavi, B. Lucht, M.J. Chon, V.B. Shenoy, P.R. Guduru, J Power Sources, 215 (2012) 145-151.

[51] U. Boesenberg, F. Meirer, Y. Liu, A.K. Shukla, R. Dell’Anna, T. Tyliszczak, G. Chen, J.C. Andrews, T.J. Richardson, R. Kostecki, J. Cabana, Chem Mater, 25 (2013) 1664-1672.

[52] G. Chen, T.J. Richardson, J Power Sources, 195 (2010) 5387-5390.

[53] M. Doyle, T.F. Fuller, J. Newman, J Electrochem Soc, 140 (1993) 1526-1533.

[54] F. Lin, D. Nordlund, T.-C. Weng, Y. Zhu, C. Ban, R.M. Richards, H.L. Xin, Nat Commun, 5 (2014).

[55] N. Balke, S. Kalnaus, N.J. Dudney, C. Daniel, S. Jesse, S.V. Kalinin, Nano Lett, 12 (2012) 33993403. 
[56] J. Liu, M. Kunz, K. Chen, N. Tamura, T.J. Richardson, J Phys Chem Lett, 1 (2010) 2120-2123.

[57] G. Brunetti, D. Robert, P. Bayle-Guillemaud, J.L. Rouvière, E.F. Rauch, J.F. Martin, J.F. Colin, F. Bertin, C. Cayron, Chem Mater, 23 (2011) 4515-4524.

[58] Y. Li, F. El Gabaly, T.R. Ferguson, R.B. Smith, N.C. Bartelt, J.D. Sugar, K.R. Fenton, D.A. Cogswell, A.L.D. Kilcoyne, T. Tyliszczak, M.Z. Bazant, W.C. Chueh, Nat Mater, 13 (2014) 1149-1156.

[59] G. Zhang, C.E. Shaffer, C.-Y. Wang, C.D. Rahn, J Electrochem Soc, 160 (2013) A610-A615.

[60] G. Zhang, C.E. Shaffer, C.-Y. Wang, C.D. Rahn, J Electrochem Soc, 160 (2013) A2299-A2305.

[61] S.-H. Ng, F. La Mantia, P. Novák, Angew Chem Int Ed, 48 (2009) 528-532.

[62] L. Cai, K. An, Z. Feng, C. Liang, S.J. Harris, J Power Sources, 236 (2013) 163-168.

[63] W.A. Paxton, Z. Zhong, T. Tsakalakos, J Power Sources, 275 (2015) 429-434.

[64] J. Nanda, J. Remillard, A. O'Neill, D. Bernardi, T. Ro, K.E. Nietering, J.-Y. Go, T.J. Miller, Adv Funct Mater, 21 (2011) 3282-3290.

[65] W. Zhao, G. Luo, C.-Y. Wang, J Power Sources, 257 (2014) 70-79.

[66] P. Maire, A. Evans, H. Kaiser, W. Scheifele, P. Novák, J Electrochem Soc, 155 (2008) A862A865.

[67] M.J. Chon, V.A. Sethuraman, A. McCormick, V. Srinivasan, P.R. Guduru, Phys Rev Lett, 107 (2011) 045503.

[68] B.P.N. Nguyen, J. Gaubicher, B. Lestriez, Electrochim Acta, 120 (2014) 319-326.

[69] Y. He, D.M. Piper, M. Gu, J.J. Travis, S.M. George, S.-H. Lee, A. Genc, L. Pullan, J. Liu, S.X. Mao, J.-G. Zhang, C. Ban, C. Wang, ACS Nano, 8 (2014) 11816-11823.

[70] J.N. Weker, N. Liu, S. Misra, J.C. Andrews, Y. Cui, M.F. Toney, Energ Environ Sci, 7 (2014) 2771-2777.

[71] D. Mazouzi, Z. Karkar, C. Reale Hernandez, P. Jimenez Manero, D. Guyomard, L. Roué, B. Lestriez, J Power Sources, 280 (2015) 533-549. 